\title{
Aortic diameter: The beginning of the end of an era
}

Alexander J. Gregory, MD, ${ }^{a}$ Elena Di Martino, PhD, ${ }^{b}$ and Paul W. M. Fedak, MD, PhD, FRCSC

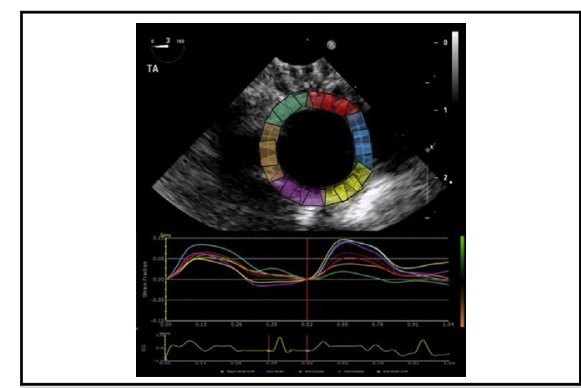

Speckle tracking software and circumferential strain analysis of the ascending aorta.

Central Message

Advances in imaging-based analysis with biomechanical models have opened a path toward new and more effective clinical tools for risk assessment of aortic aneurysms.

See Article page 503. understanding is facilitated by collaborative efforts between clinicians and bioengineers with biomechanical computational models. ${ }^{1}$ Moreover, advances in cardiovascular imaging techniques, coupled with the exponential growth of computing power, have presented a unique opportunity for noninvasive assessment of aortic biomechanics in our patients, allowing the creation of more robust treatment protocols. ${ }^{2}$ Despite these promising developments, a critical gap remains. How can we leverage our growing knowledge of aortic biology and mechanics to improve clinical outcomes? Can we use novel imaging metrics that reflect aortic biomechanics to move beyond aortic diameter as the touchstone of our clinical strategies for prophylactic surgical resection of aortic aneurysms?

The study in this issue of the Journal by Emmott and colleagues ${ }^{3}$ represents an important step toward bridging this gap. Echocardiography is noninvasive, widely available, and relatively inexpensive. Emmott and colleagues ${ }^{3}$ applied speckle tracking analysis to echocardiographic images acquired in the operating room, generating comprehensive metrics of aortic mechanics in patients with aneurysmal disease. Surgically resected tissue was then subjected to biomechanical testing and histologic analysis. In addition to quantifying stiffness, the investigators use a less familiar concept of "energy loss," which in part reflects the viscoelasticity of the aortic wall altered by shear forces at the blood-tissue interface. ${ }^{4}$ Importantly, the study confirms that echocardiography can provide noninvasive metrics in human patients that correlate with altered biomechanical properties and disrupted histopathologic characteristics of the proximal aorta. Linking imaging-based measures of in vivo aortic properties with ex vivo mechanical and

cellular markers of pathology is critical to the discovery and validation of novel tools for individualized patient decision strategies.

What does the future hold for these emerging technologies? The optimal use and limitations of the various imaging modalities, such as computed tomography, magnetic resonance imaging, and ultrasonography, will need to be delineated. Other translational hurdles will be faced. Applying biomechanical models in the clinical arena will force us to accept certain biophysical assumptions on which they are based. Informed, transparent, and constructive debate within the aortic scientific community should be encouraged as we decipher which assumptions and models are the most accurate. Conversely, the search for "biologic and pathophysiologic truth" will need to be balanced with pragmatism and an eye on the final goal: development of biomechanical markers that best predict future aortic events.

Although the road ahead is filled with many unanswered questions, this study provides an encouraging proof of concept that noninvasive biomechanical assessments may help guide resection strategies at the point of care. But it is just the start. Large databases will be needed to correlate biomechanical data with clinical outcomes. Strong collaborative relationships between experts in cardiovascular surgery, medical imaging, physiology, and bioengineering will be essential as we move toward development of surgical risk assessments beyond aneurysm size alone. Aortic diameter-the end is near. 


\section{References}

1. Martufi G, Forneris A, Appoo JJ, Di Martino ES. Is there a role for biomechanical engineering in helping to elucidate the risk profile of the thoracic aorta? Ann Thorac Surg. 2016;101:390-8.

2. Guzzardi DG, Barker AJ, van Ooij P, Malaisrie SC, Puthumana JJ, Belke DD, et al. Valve-related hemodynamics mediate human bicuspid aortopathy: insights from wall shear stress mapping. J Am Coll Cardiol. 2015;66: 892-900.

3. Emmott A, Alzahrani H, Alreshidan M, Therrien J, Leask RL, Lachapelle K Transesophageal echocardiographic strain imaging predicts aortic biomechanics: Beyond diameter. J Thorac Cardiovasc Surg. 2018;156:503-12.

4. Roca F, Iacob M, Remy-Jouet I, Bellien J, Joannides R. Evidence for a role of vascular endothelium in the control of arterial wall viscosity in humans. Hypertension. 2018;71:143-50.

5. Fedak PW, Verma S. Bicuspid aortopathy and the development of individualized resection strategies. J Thorac Cardiovasc Surg. 2014;148:2080-1. 